\title{
Report of the Working Group for Dietary Salt Reduction of the Japanese Society of Hypertension: (2) Assessment of Salt Intake in the Management of Hypertension
}

\author{
Yuhei KAWANO ${ }^{1)}$, Takuya TSUCHIHASHI ${ }^{2)}$, Hideo MATSUURA ${ }^{3)}$, Katsuyuki ANDO ${ }^{4}$, \\ Toshiro FUJITA ${ }^{4)}$, and Hirotsugu UESHIMA ${ }^{5)}$
}

\begin{abstract}
Restriction of dietary salt is widely recommended in the management of hypertension, but assessment of individual salt intake has drawn little attention. The understanding of salt intake is important as a guide for optimizing salt-restriction strategies. However, precise evaluation of salt intake is difficult. More reliable methods are more difficult to perform, whereas easier methods are less reliable. Thus, the method to assess salt intake should be determined as the situation demands. The Working Group for Dietary Salt Reduction of the Japanese Society of Hypertension recommends the assessment of individual salt intake using one of the following methods in the management of hypertension. 1) The measurement of the sodium (Na) excretion from 24-h urine sampling or nutritionist's analysis of the dietary contents, which are reliable but difficult to perform, are suitable for facilities specializing in the treatment of hypertension. 2) Estimation of the $\mathrm{Na}$ excretion from the $\mathrm{Na}$ /creatinine $(\mathrm{Cr})$ ratio in spot urine is less reliable but practical and is suitable for general medical facilities. 3) Estimation using an electronic salt sensor equipped with a calculation formula is also less reliable but is simple enough that patients can use it themselves. The patients are considered to be compliant with the salt-restriction regimen if salt intake measured by whichever method is less than $6 \mathrm{~g}$ (100 mmol)/day. (Hypertens Res 2007; 30: 887-893)
\end{abstract}

Key Words: salt intake, food weighing, food questionnaire, urinary sodium excretion, hypertension

\section{Introduction}

Excessive salt or sodium $(\mathrm{Na})$ intake causes hypertension, and restriction of salt intake is widely recommended for the management of hypertension. In the 2004 version of the Japanese Society of Hypertension (JSH) Guidelines for the Management of Hypertension (JSH 2004), the target of salt restriction was tightened from $7 \mathrm{~g} /$ day or less to less than $6 \mathrm{~g} /$ day (1). On the other hand, while the salt intake in Japan is decreasing, it is still high, being about $11 \mathrm{~g}$ /day (2). Also, salt intake shows considerable individual variation and daily fluctuation in the same individual.

An understanding of individual salt intake is considered to be important for successful salt reduction, because it leads to appropriate guidance and judgement of whether the target of salt restriction has been attained. However, there are several problems with the assessment of salt intake, and its imple-

From the ${ }^{1}$ Division of Hypertension and Nephrology, Department of Medicine, National Cardiovascular Center, Suita, Japan; ${ }^{2}$ Division of Hypertension, National Kyushu Medical Center, Fukuoka, Japan; ${ }^{3}$ Department of Internal Medicine, Saiseikai Kure Hospital, Kure, Japan; ${ }^{4}$ Department of Internal Medicine, Faculty of Medicine, University of Tokyo, Tokyo, Japan; and ${ }^{5}$ Department of Health Science, Shiga University of Medical Science, Otsu, Japan.

Address for Reprints: Yuhei Kawano, M.D., Ph.D., Division of Hypertension and Nephrology, Department of Medicine, National Cardiovascular Center, 5-7-1 Fujishirodai, Suita 565-8565, Japan. E-mail: ykawano@hsp.ncvc.go.jp

Received June 5, 2007; Accepted in revised form July 8, 2007. 
Table 1. Evaluation Methods of Salt Intake

\begin{tabular}{lcc}
\multicolumn{1}{c}{ Evaluation method } & Reliability & Convenience \\
\hline Evaluations based on dietary contents & & \\
Weighing method & $\bigcirc$ & $\times$ \\
Questionnaire method & $\bigcirc$ & $\triangle$ \\
Measurement before intake & $\bigcirc$ & $\bigcirc$ \\
Evaluation using test paper or salt sensor & $\times$ & $\times$ \\
Evaluations based on the measurement of urinary Na excretion & $\bigcirc$ & $\triangle$ \\
24-h pooled urine & $\bigcirc$ & $\triangle$ \\
Nighttime or early morning urine & $\bigcirc$ & $\bigcirc$ \\
The second urine sample after waking & $\triangle\left(\bigcirc^{*}\right)$ & $\bigcirc$ \\
Spot urine & $\times\left(\triangle \triangle^{* *}\right)$ & \\
Evaluation using test paper or salt sensor & & \\
\hline
\end{tabular}

(O), excellent; $\bigcirc$, good; $\triangle$, fair; $\times$, poor. *When a formula for the estimation of the daily creatinine $(\mathrm{Cr})$ excretion is used. **When a salt sensor installed with the formula is used.

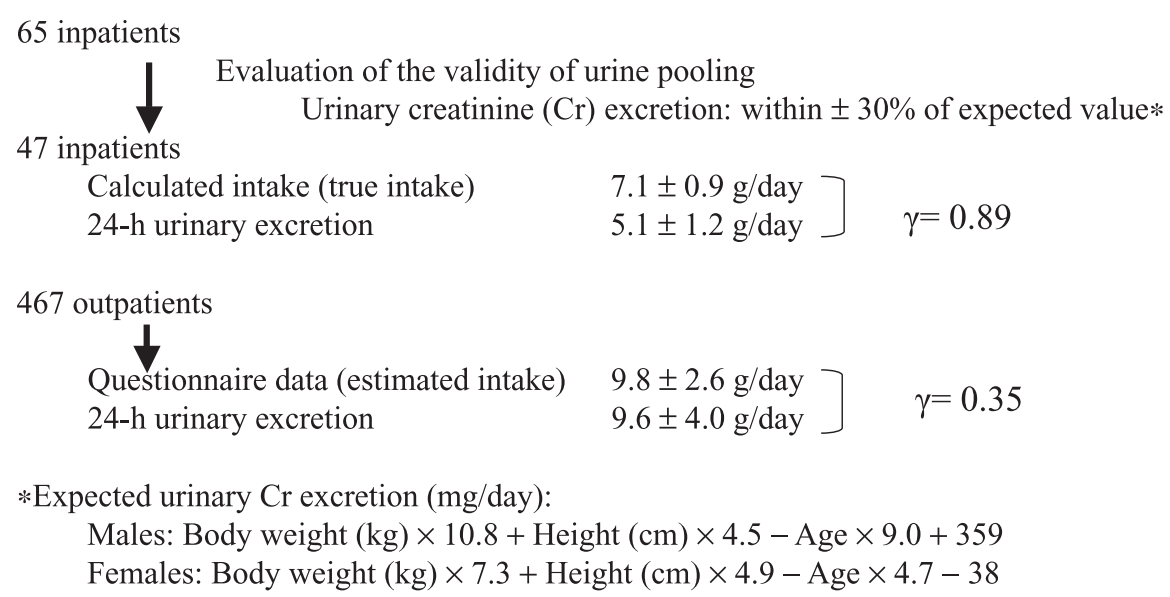

Fig. 1. Calculated dietary salt intake and 24-h urinary excretion in inpatients and estimated salt intake based on a questionnaire and 24-h urinary excretion in outpatients (from data of Fukumoto et al. (9))

mentation is often difficult. Most of the current guidelines for the management of hypertension do not mention the methodological aspect of assessing salt intake. While the guidelines of the World Health Organization and International Society of Hypertension (WHO/ISH) state that counseling by a skilled nutritionist and monitoring of the urinary $\mathrm{Na}$ level are necessary in most cases, they do not mention specific methods for these purposes (3).

This report describes variations and characteristics of salt intake-assessment methods and proposes the guidelines for the assessment of salt intake for the management of hypertension as part of the activities of the Working Group for Dietary Salt Reduction of the JSH. The Japanese version of the working group report has been published previously (4).

\section{Methods to Assess Salt Intake}

There are several methods for the assessment of salt intake. In general, however, the choice of method involves a compromise between accuracy and ease-of-use, with relatively precise methods being difficult to perform, and simpler methods being less reliable (Table 1). Also, because salt intake is not fixed in each person, its assessment is naturally subject to limitations in accuracy (5). Sodium, which is important in the occurrence and progression of hypertension, is primarily ingested as salt $(\mathrm{NaCl})$. Since $1 \mathrm{~g}$ of salt is equivalent to 17 $\mathrm{mmol}(17 \mathrm{mEq})$ of Na, $6 \mathrm{~g}$ of salt is about $100 \mathrm{mmol}(100$ $\mathrm{mEq}$ ) of $\mathrm{Na}$. In terms of relative weight, a given amount of $\mathrm{Na}$ in salt would weigh 2.5 times more than the equivalent amount as pure $\mathrm{Na}$ (for example, $400 \mathrm{mg}$ of $\mathrm{Na}$ is equal to 1 $\mathrm{g}$ of salt).

\section{Assessment Based on Dietary Contents}

Weighing Method

This method, by which salt intake is estimated by weighing 
Table 2. Formula for the Estimation of the 24-h Sodium (Na) Excretion from Nighttime Urine Data and Estimated Cr Excretion (18)

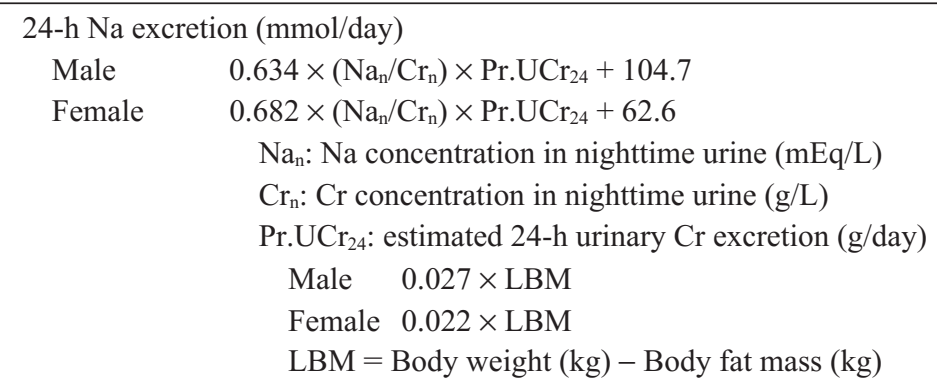

Cr, creatinine; LBM, lean body mass.

Table 3. Formula for the Estimation of the 24-h Na Excretion from Data in the Second Urine Sample after Waking and Estimated Cr Excretion (19)

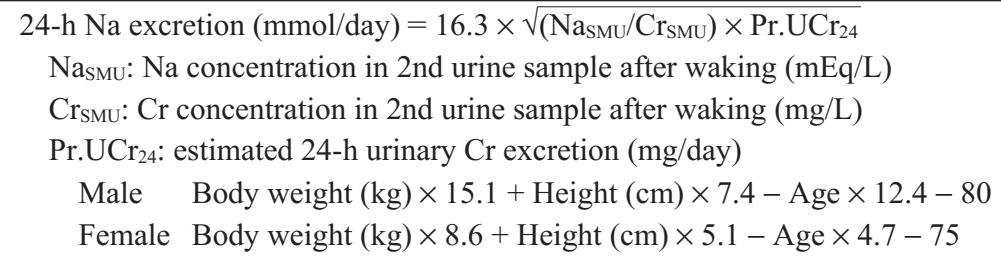

Cr, creatinine.

the food ingested by each subject, is highly reliable ( 6 ). Concerning $\mathrm{Na}$, the values estimated from the food weight based on the Standard Tables of Food Composition in Japan (7) have been shown to be close to, and strongly correlated with, the actual values measured in the ingested food. However, this method is complicated and requires calculation by a nutritionist. Also, a 1-day survey is considered to be insufficient for accurate assessment of salt intake, which changes from day to day.

\section{Questionnaire Method}

By this method, dietary salt intake is estimated from data obtained by a questionnaire or interview performed over one to several days. While it is easier than the weighing method, this method still requires calculation by a nutritionist. Although there has been a report suggesting that its reliability is comparable to that of the weighing method (8), its accuracy is considered to be slightly inferior. Also, while the mean salt intake estimated by interview has been reported to agree with the value based on the 24-h urinary $\mathrm{Na}$ excretion, its correlation to actual salt intake was not high, and actual salt intake may be underestimated by this method (9) (Fig. 1).

\section{Measurement before Intake}

In the measurement-before-intake method, daily salt intake is determined by measuring or estimating the salt content of food to be eaten before ingestion. If performed precisely, this method is highly reliable. Hospital meals and test meals for clinical research are examined by this method. Since the salt intake is revealed before ingestion of the meal, this method is useful for the practice of salt reduction. However, it is inconvenient to measure the salt content before each meal. Moreover, accurate determination requires calculation by a nutritionist, although rough calculation can be performed by untrained individuals.

\section{Measurement of the Urinary Na Excretion}

\section{Measurement by 24-h Urine}

In this method, urine is collected for $24 \mathrm{~h}$, and salt intake is assessed by determining the urinary $\mathrm{Na}$ excretion. This method is considered to be reliable and is used in many clinical and epidemiological studies, including the international cooperative Intersalt study (10). However, it is relatively difficult to perform because of the necessity of 24-h urine sampling, and inadequate urine pooling leads to underestimation of salt intake. The nuisance of 24-h urine collection is slightly mitigated by the use of a portable urine sampler (Urinmate ${ }^{\circledR}$ ), which allows fractionated partial urine sampling (11). For accurate assessment of salt intake, even the 24-h urine sampling method is insufficient if performed over only 1 day, and thus measurement over several days is considered necessary $(11,12)$. In addition, while most of the ingested $\mathrm{Na}$ is excreted in urine, part of it is contained in feces or sweat. Salt intake determined from the $\mathrm{Na}$ excretion in 24-h urine has been shown to be $0.5-3 \mathrm{~g}$ /day lower than the true intake, and 


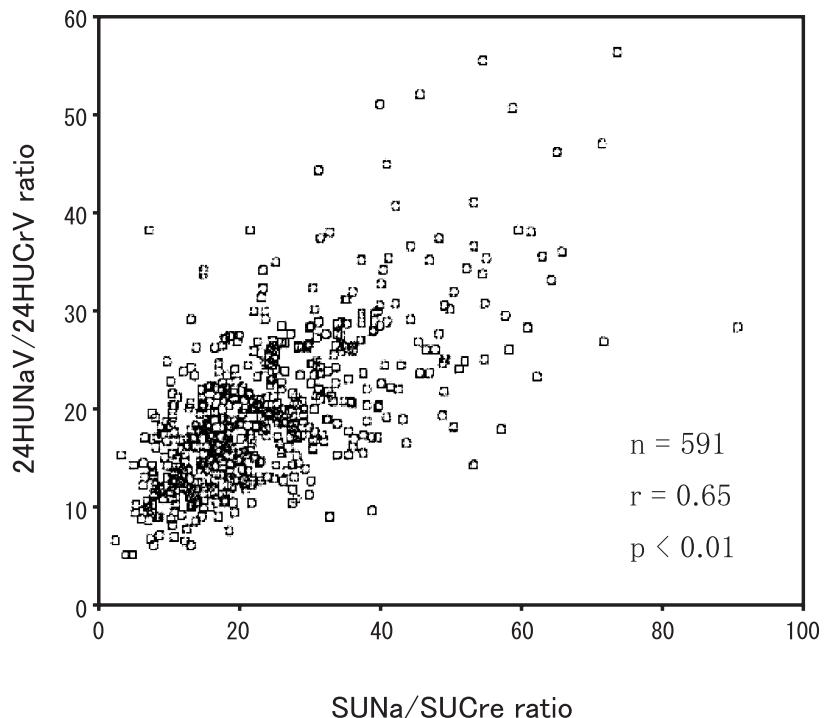

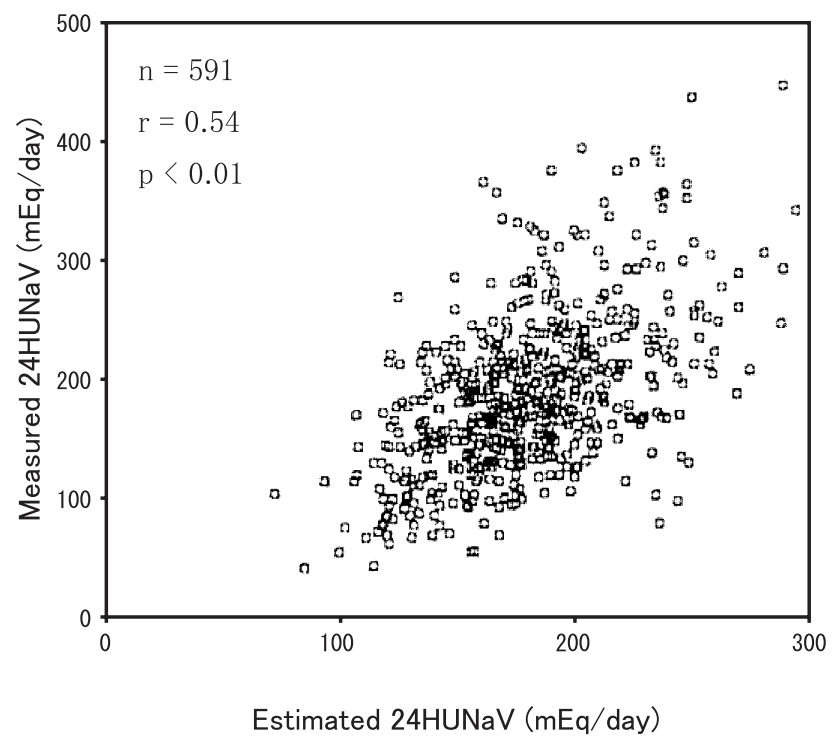

Evaluated in the 591 Japanese (aged 20-59 years) who participated in the Intersalt Study

24-h urinary salt excretion $=21.98 \times(\mathrm{Na} / \mathrm{Cr} \text { in spot urine } \times \text { expected } 24-\mathrm{h} \mathrm{Cr} \text { excretion })^{0.392}$

Fig. 2. Evaluation of salt intake by spot urine. The left plot shows the relationship between the sodium (Na)/Cr ratio in spot urine (SUNa/SUCr ratio) and $\mathrm{Na} / \mathrm{Cr}$ ratio in 24-h urine (24HUNaV/24HUCrV ratio). The right plot shows the relationship between the estimated 24-h urinary Na excretion by the calculation formula based on spot urine data (Estimated 24HUNaV) and measured 24-h urinary Na excretion (Measured 24HUNaV) (from Tanaka et al. (21) with modification).

Table 4. Formula for the Estimation of the 24-h Na Excretion from Spot Urine Data and Estimated Cr Excretion (21)

Cr, creatinine.

it is underestimated even with complete urine collection $(9$, 12, 13) (Fig. 1).

\section{Measurement by Nighttime and Overnight Urine}

Sampling of nighttime or early morning (overnight) urine, which consists of nighttime urine, is often employed, because it is easier than 24-h urine sampling and still provides a relatively long-term sample. In addition, $\mathrm{Na}$ excretion in nighttime urine is well correlated with that in 24-h urine $(14,15)$. However, $\mathrm{Na}$ excretion exhibits diurnal fluctuation, being about $20 \%$ lower during the nighttime than the daytime (16, 17). Therefore, simple estimation of salt intake from the $\mathrm{Na}$ excretion in nighttime urine is considered to result in greater underestimation than that in 24-h collected urine. However, the 24-h Na excretion estimated by the following calculation using $\mathrm{Na}$ excretions in nighttime urine has been reported to be in relatively close agreement with the value determined in 24h sampled urine (18) (Table 2). In this method, 24-h Na excretion was estimated by applying $\mathrm{Na}$ and creatinine $(\mathrm{Cr})$ excretions in nighttime urine and estimated 24-h urinary $\mathrm{Cr}$ excretion, calculated using the lean body mass from the height, body weight, and body fat mass.

\section{Measurement by the Second Urine Sample after Waking}

In another previously reported method to estimate the daily urinary $\mathrm{Na}$ excretion, the $\mathrm{Na}$ and $\mathrm{Cr}$ concentrations in the second urine sample after waking, and the 24-h urinary Cr excretion estimated from height, body weight, and age, are applied to a calculation formula (19) (Table 3 ). The Na excretion estimated by this method is closely correlated with the value determined in 24-h pooled urine. However, its clinical use may be limited by the condition that the urine must be col- 
Table 5. Guidelines for the Evaluation of Salt Intake

\begin{tabular}{|c|c|c|}
\hline Evaluation method & Recommendability & Major application target \\
\hline $\begin{array}{l}\text { Measurement of the Na excretion in } 24-\mathrm{h} \\
\text { pooled urine, or Weighing or questionnaire } \\
\text { survey by a nutritionist }\end{array}$ & $\begin{array}{l}\text { Although highly reliable and recommend- } \\
\text { able, these methods are complicated. Rec- } \\
\text { ommended if the patients' cooperation and } \\
\text { the facility's ability are secured }\end{array}$ & $\begin{array}{l}\text { Special facilities for hypertension treat- } \\
\text { ment }\end{array}$ \\
\hline $\begin{array}{l}\text { Estimation as } \mathrm{Na} / \mathrm{Cr} \text { ratio based on mea- } \\
\text { surement of } \mathrm{Na} \text { and } \mathrm{Cr} \text { in spot urine sam- } \\
\text { ples* }\end{array}$ & $\begin{array}{l}\text { Although the reliability is relatively low, } \\
\text { the method is simple and recommended as } \\
\text { a practical evaluation procedure }\end{array}$ & Medical facilities in general \\
\hline $\begin{array}{l}\text { Estimation in early morning urine (night- } \\
\text { time urine) using an electronic salt sensor } \\
\text { installed with calculation formula** }\end{array}$ & $\begin{array}{l}\text { Although the reliability is relatively low, } \\
\text { the method is recommendable. It is conve- } \\
\text { nient and can be performed by the patients } \\
\text { themselves }\end{array}$ & Patients themselves \\
\hline
\end{tabular}

lected as the second urine sample after waking and before breakfast.

\section{Measurement by Spot Urine}

Evaluation of salt intake using a spot urine sample collected at any time would be easy to perform. The $\mathrm{Na}$ excretion per amount of $\mathrm{Cr}$ in spot urine correlates relatively well with the $\mathrm{Na}$ excretion per amount of $\mathrm{Cr}$ in 24-h urine sampling $(20,21)$ (Fig. 2), but the correlation between the $\mathrm{Na}$ excretion in spot urine and that in 24-h pooled urine is not very high $(15,20)$. However, the estimated $\mathrm{Na}$ excretion calculated using a formula incorporating the estimated 24-h urinary $\mathrm{Cr}$ excretion (Table 4) is reportedly close to the actually measured 24-h urinary Na excretion (21) (Fig. 2). The method to estimate the daily $\mathrm{Na}$ intake from the $\mathrm{Na}$ excretion per gram of $\mathrm{Cr}$ calculated from the $\mathrm{Na}$ and $\mathrm{Cr}$ concentrations in spot urine is not very reliable but is simple and considered to be clinically useful.

\section{Assessment Using Test Paper or a Salt Sensor}

This method, by which salt intake is estimated by measuring the salt concentration in spot urine or overnight urine using test paper or an electronic salt sensor, is the simplest $(22,23)$. The test paper or salt sensor usually detects chloride $(\mathrm{Cl})$ rather than $\mathrm{Na}$, and the results of examination of overnight urine using a test paper have been shown to be correlated with salt intake estimated by a nutritional survey (23). However, these should be regarded as unreliable and semi-quantitative methods. Recently, a urinary salt sensor, which estimates salt intake by analyzing data in overnight urine using a preinstalled calculation formula, has become available and is expected to increase the reliability (24).

The salt concentration in food can be determined using test paper or a salt sensor. In one previous report, however, the salt concentration of miso soup was found to be unrelated to the urinary salt level (23). The estimation of daily salt intake from the salt concentration of a single food item is thus considered to be difficult.

\section{Assessment of Salt Intake for the Management of Hypertension}

As mentioned above, there are several problems with the assessment of salt intake. Even measurement of the dietary salt content and the 24-h urine sampling method, which are considered to be highly reliable, are not sufficiently accurate and are difficult to perform (Table 1). Although the examination of the $\mathrm{Na} / \mathrm{Cr}$ ratio in spot urine and the test paper method are easier to perform, they are less reliable. Calculation using a formula and the data of nighttime or spot urine is more reliable but more complicated. Also, it should be noted that salt intake determined from the urinary $\mathrm{Na}$ excretion or by the questionnaire method tends to be underestimated.

Despite these problems, the assessment of salt intake in individual patients is useful for motivating patients to reduce their salt intake, as well as for guiding their progress and evaluating the results. Such assessment is strongly recommended for the management of hypertension, because it provides patients with concrete numerical values of their salt intake. The use of more reliable methods is desirable, if possible, but even less reliable methods are of clinical value.

The Working Group for Dietary Salt Reduction of the JSH proposes the guidelines shown in Table 5 for the assessment of salt intake for the management of hypertension. In the management of hypertensive patients, salt intake should be evaluated individually using one of the following methods whenever possible.

1) The measurement of the $\mathrm{Na}$ excretion in 24-h pooled urine or a nutritionist's analysis of the dietary contents: These 
methods are reliable but often difficult to perform. They are recommended depending on the patients' cooperativeness and the facility's competence and are suited for facilities specializing in hypertension.

2) Estimation of the $\mathrm{Na}$ excretion from the $\mathrm{Na} / \mathrm{Cr}$ ratio in spot urine: This method is less reliable, but it is easy to perform and is considered to be practical. Since the daily $\mathrm{Cr}$ excretion of Japanese is about $1 \mathrm{~g}$ (about $10 \mathrm{mmol}$ ) (10), salt intake is estimated to be about $6 \mathrm{~g}$ if the $\mathrm{Na}$ excretion per gram of $\mathrm{Cr}$ is $100 \mathrm{mmol}$. Therefore, this method is considered to be useful for salt reduction guidance. However, the urinary $\mathrm{Cr}$ excretion varies considerably according to the physique, age, and gender of patients. Therefore, note that true salt intake is lower in small females and higher in large males than the value estimated from the $\mathrm{Na} / \mathrm{Cr}$ ratio. Overnight urine (nighttime urine) may also be used. The reliability can be increased by the use of a calculation formula (Tables 2-4).

3) Estimation using an electronic salt sensor equipped with a calculation formula in early morning urine (overnight urine): Although this method is less reliable, it can be recommended, because it is simple and can be performed by the patients themselves. However, the patient must purchase a salt sensor, or the medical facility must lend one to the patient, for home monitoring.

According to the guidelines for lifestyle modifications in the management of hypertension, the patient is considered to be compliant with the salt restriction regimen if the salt intake measured by whichever method is less than $6 \mathrm{~g} \mathrm{(100} \mathrm{mmol}$ $\mathrm{Na}$ /day and not if it is higher.

\section{Conclusions}

Although there are several methods for the assessment of salt intake, the precise determination of salt intake in individual patients is difficult. Reliable methods are difficult to perform, and simpler methods are less reliable. However, the assessment of salt intake is strongly recommended, because it is useful for informing patients of their salt intake and conducting salt restriction.

In the management of hypertension, it is desirable to assess salt intake by one of the following three methods whenever possible: 1) Measurement of the Na excretion in 24-h pooled urine or a nutritionist's analysis of the dietary contents: Although these are desirable methods because of their reliability, they are difficult to perform and are suited for facilities specializing in hypertension. 2) Estimation of the $\mathrm{Na}$ excretion from the $\mathrm{Na} / \mathrm{Cr}$ ratio in spot urine: This is a less reliable but practical method and is suited for general medical facilities. Overnight urine (nighttime urine) may also be used, and the reliability is increased by the use of a calculation formula. 3) Estimation using an electronic salt sensor equipped with a calculation formula in overnight urine: While this method is less reliable, it can be recommended, because it is simple and can be performed by the patients themselves. The patient is judged to be compliant with the salt restriction reg- imen if salt intake (excretion) estimated by any of the methods is less than $6 \mathrm{~g}(100 \mathrm{mmol}) /$ day but not if it is higher.

\section{References}

1. Japanese Society of Hypertension: Japanese Society of Hypertension Guidelines for the Management of Hypertension (JSH 2004). Hypertens Res 2006; 29 (Suppl): S1S105.

2. Lifestyle-Related Disease Control Section, General Affairs Division, Health Bureau, Ministry of Health, Labor and Welfare: An Outline of the Results of the 2004 National Health and Nutrition Survey (in Japanese). http:// www.mhlw.go.jp/houdou/2006/05/h0508-1a.html

3. Guidelines Subcommittee: 1999 World Health Organization-International Society of Hypertension Guidelines for the management of hypertension. J Hypertens 1999; 17: 151-183.

4. Kawano Y, Tsuchihashi T, Matsuura H, Ando K, Fujita T, Ueshima H: Assessment of salt intake in the management of hypertension, in Report of the Working Group for Dietary Salt Reduction of the Japanese Society of Hypertension. Tokyo, Japanese Society of Hypertension, 2006, pp 13-23 (in Japanese).

5. Liu K, Cooper R, McKeever J, et al: Assessment of the association between habitual salt intake and high blood pressure: methodological problems. Am J Epidemiol 1979; 110: 219-226.

6. Saito T, Shimizu A, Hokimoto S, et al: Salt intake of the Japanese. J Kyoto Women's Univ Food Sci Soc 1995; 50: 20-27 (in Japanese).

7. Yoshita K, Miura K, Okayama A, et al: A validation study on food composition tables for the international cooperative INTERMAP study in Japan. Environ Health Prev Med 2005; 10: 150-156.

8. Takagi H, Kaneko S, Saeki K, et al: Estimation of salt intake using a questionnaire. J Health Human Ecol 1993; 59: 113-122 (in Japanese).

9. Fukumoto T, Ueno M, Haga K, et al: Estimation of salt intake at home. Lecture Summaries at the 55th Conference of the Japanese Society of Nutrition and Food Science. Tokyo, Japanese Society of Nutrition and Food Science, 2001, p 212 (in Japanese).

10. Intersalt Cooperative Research Group: Intersalt: an international study of electrolyte excretion and blood pressure. Results for 24 hour urinary sodium and potassium excretion. BMJ 1998; 297: 319-328.

11. Yamori Y, Nara Y, Kihara M, et al: Simple method for sampling consecutive 24-hour urine for epidemiological and clinical studies. Clin Exp Hypertens 1984; 6: 11611167.

12. Luft FC, Fineberg NS, Sloan RS: Estimating dietary sodium intake in individuals receiving a randomly fluctuating intake. Hypertension 1982; 4: 805-808.

13. Sakai H, Ikeda T, Saito H: Effects of seasons and perspiration on $\mathrm{Na}$ and protein intake and their daily urinary excretion. Jpn J Public Health 1989; 36: 309-315 (in Japanese).

14. Liu K, Dyer AR, Cooper RS, et al: Can overnight urine replace 24-hour urine collection to assess salt intake? 
Hypertension 1979; 1: 529-536.

15. Tsai TJ, Chen YM, Hsieh BS, et al: Comparison between spot urine and overnight urine in the estimation of 24-hour excretion of urine protein, sodium and kallikrein. $J$ Formos Med Assoc 1991; 90: 755-759.

16. Kawano Y, Kawasaki T, Kawazoe N, et al: Circadian variations of urinary dopamine, norepinephrine, epinephrine and sodium in normotensive and hypertensive subjects. Nephron 1990; 55: 277-282.

17. Staessen J, Broughton PMG, Fletcher AE, et al: The assessment of the relationship between blood pressure and sodium intake using whole-day, daytime and overnight urine collections. J Hypertens 1991; 9: 1035-1040.

18. Kamata K, Tochikubo O: Estimation of 24-h urinary sodium excretion using lean body mass and overnight urine collected by a pipe-sampling method. J Hypertens 2002; 20: 2191-2197.

19. Kawasaki $\mathrm{T}$, Itoh $\mathrm{K}$, Uezono $\mathrm{K}$, et al: A simple method for estimating $24 \mathrm{~h}$ urinary sodium and potassium excretion from second morning voiding urine specimen in adults. Clin
Exp Pharmacol Physiol 1993; 20: 7-14.

20. Milne FJ, Gear JSS, Laidley L, et al: Spot urinary electrolyte concentrations and 24 hour excretion. Lancet 1980; 2: 1135.

21. Tanaka T, Okamura T, Miura K, et al: A simple method to estimate populational 24-h urinary sodium and potassium excretion using a casual urine specimen. J Hum Hypertens 2002; 16: 97-103.

22. Luft FC, Aronoff GR, Sloan RS, et al: The efficacy of quantitative and qualitative chloride titrators in the estimation of human salt intake. Klin Wochenschr 1985; 63: 62-67.

23. Tabata S, Onishi T, Nakamura H, et al: Evaluation of the measurement of the urinary salt concentration using Salt Paper in health screening. Hokuriku J Public Health 1987; 14: 42-46 (in Japanese).

24. Yamasue K, Tochikubo O, Kono E, et al: Self-monitoring of home blood pressure with estimation of daily salt intake using a new electrical device. J Hum Hypertens 2006; 20: 593-598. 